\title{
Incidência de pericardite pós COVID-19 em pacientes de uma clínica cardiológica, no período de março a junho de 2020
}

\author{
Incidence of post COVID-19 pericarditis in patients of a cardiological clinic, in the period \\ from march to june 2020
}

Incidencia de pericarditis post-COVID-19 en pacientes de una clínica cardiológica, en el período de marzo a junio de 2020

Camila Guerreiro Bentes ${ }^{1 *}$, Mariana Diniz Araújo1, Maria Elizabeth Navegantes Caetano², Vítor Bruno Teixeira de Holanda ${ }^{2}$.

\section{RESUMO}

Objetivo: Identificar a incidência de pericardite pós- COVID-19 em pacientes atendidos em uma clínica cardiológica, bem como discutir os fatores de risco e possíveis terapêuticas. Métodos: Foi realizada coleta de dados através do protocolo de pesquisa em prontuários de pacientes atendidos na referida clínica, buscando identificar os pacientes de tiveram COVID-19 e destes quais evoluíram com pericardite. Todos os dados foram tabelados utilizando-se o software Microsoft Excel e análise estatística realizada no programa Biostat 5.0. Resultados: Foram encontrados 4 pacientes evoluindo com pericardite aguda pós COVID-19, sendo observada incidência de $11,76 \%$. A maioria dos pacientes apresentou comorbidades cardiocerebrovasculares, que sabidamente estão associadas a pior prognóstico e morbimortalidade na infecção pelo SARS-CoV-2. Todos os pacientes apresentaram desfecho clínico favorável, após tratamento com dose terapêutica usual com colchicina. Conclusão: Conclui-se que apesar de complicação tardia da COVID-19, a pericardite aguda na casuística do estudo, manteve seu padrão autolimitado, assim como sua relevante taxa de incidência assegura as manifestações cardíacas como uma das principais e mais importantes dentre as manifestações extrapulmonares da COVID-19.

Palavras-chave: COVID-19, SARS-CoV-2, Pericardite.

\begin{abstract}
Objective: This present study aims to identify the incidence of post-COVID-19 pericarditis in patients seen at a cardiology clinic, as well as to discuss risk factors and possible therapies. Methods: Data collection was performed through the research protocol on medical records of patients seen at the clinic, seeking to identify patients with COVID-19 and those who developed pericarditis. All data were tabulated using Microsoft Excel software and statistical analysis performed using the Biostat 5.0 program. Results: In our study, we found 4 patients who evolved with acute pericarditis after COVID-19, with an incidence of $11.76 \%$. Most patients had cardiocerebrovascular comorbidities, which are known to be associated with a worse prognosis and morbidity and mortality in SARS-CoV-2 infection. All patients had a favorable clinical outcome after treatment with the usual therapeutic dose with colchicine. Conclusion: It is concluded that despite the late complication of COVID-19, acute pericarditis in the study series, maintained its self-limited pattern, as well as its relevant incidence rate ensures cardiac manifestations as one of the main and most impotent of COVID-19 extrapulmonary manifestations.
\end{abstract}

Keywords: COVID-19, SARS-CoV-2, Pericarditis.

\section{RESUMEN}

Objetivo: Identificar la incidencia de pericarditis post-COVID-19 en pacientes atendidos en una clínica de cardiología, así como discutir factores de riesgo y posibles terapias. Métodos: La recolección de datos se realizó a través del protocolo de investigación en las historias clínicas de los pacientes atendidos en la clínica, buscando identificar a los pacientes con COVID-19 y los que desarrollaron pericarditis. Todos los datos se

${ }^{1}$ Fundação Santa Casa de Misericórdia do Pará (FSCMPA), Belém - PA.

*E-mail: camilagbentes@hotmail.com

2 Clínica Cardiodiagnóstico, Belém - PA. 
tabularon utilizando el software Microsoft Excel y el análisis estadístico se realizó utilizando el programa Biostat 5.0. Resultados: Se encontraron cuatro pacientes que evolucionaron con pericarditis aguda posterior al COVID-19, con una incidencia del 11,76\%. La mayoría de los pacientes tenían comorbilidades cardiocerebrovasculares, que se sabe que se asocian con un peor pronóstico, morbilidad y mortalidad en la infección por SARS-CoV-2. Todos los pacientes tuvieron una evolución clínica favorable después del tratamiento con la dosis terapéutica habitual de colchicina. Conclusión: Se concluye que a pesar de la complicación tardía del COVID-19, la pericarditis aguda en la serie de estudio mantuvo su patrón autolimitado, así como su tasa de incidencia relevante asegura las manifestaciones cardíacas como una de las principales y más importantes entre las manifestaciones extrapulmonares de COVID-19.

Palabras clave: COVID-19, SARS-CoV-2, Pericarditis.

\section{INTRODUÇÃO}

Em dezembro de 2019, na cidade de Wuhan, na China, emergiu uma nova variedade de coronavírus, o betacoronavírus envelopado de RNA, denominado Síndrome Respiratória Aguda Severa Coronavírus - 2 (SARS-CoV-2), causador da doença pelo coronavírus 2019 (COVID-19). Desde seu surgimento a COVID-19 espalhou-se, sendo em março de 2020 declarada como pandemia pela Organização Mundial de Saúde (OMS) apresentando importantes impactos econômicos e de saúde globalmente (MERAD M e MARTIN J, 2020; MOORE JB e JUNE CH 2020; SOUSA MRN, et al., 2020).

Os coronavírus são compostos por 4 gêneros da subfamília Orthocoronavirinae, chamados alfa, beta, que infectam mamíferos e gama e delta, que infectam aves. O SARS-CoV-2 é o terceiro betacoronavírus que infecta humanos, sua estrutura envelopada é composta por uma fita-simples de RNA. A ligação deste vírus com as células alvo ocorre através da glicoproteína Spike (proteína S), que media a ligação com uma grande variedade de receptores transmembrana, incluindo o receptor de ECA II, uma proteína expressa em diversos tecidos, especialmente nas células alveolares tipo II nos pulmões, rins, intestino e coração (GUZIK TJ, et al., 2020; MERHA MR, et al., 2020). Essa ligação desencadeia efeitos deletérios da ativação do sistema reninaangiotensina-aldosterona, tais como vasoconstrição, alteração da permeabilidade vascular, remodelamento cardíaco e injúria pulmonar aguda (FIGUEIREDO NETO JA, et al., 2020).

A infecção por SARS-CoV-2 provoca uma doença de amplo espectro, podendo apresentar-se de forma assintomática até falência respiratória severa, lesão miocárdica intensa e consequente morte. As principais manifestações clínicas provocadas pela doença incluem caracteristicamente sintomas do trato respiratório, como tosse seca, podendo ainda evoluir com complicações relacionadas a síndrome do desconforto respiratório agudo (SARA) (YUKI K, et al., 2020).

O acometimento cardíaco é uma característica proeminente na infecção por SARS-CoV-2 sendo associada a pior prognóstico. Até $30 \%$ dos pacientes internados infectados pelo vírus podem apresentar manifestações miocárdicas, representadas principalmente por níveis elevados de troponina I (MITRANI RD, et al., 2020). A fisiopatogenia da lesão provocada pelo SARS-CoV-2 no miocárdio ainda não é completamente conhecida, contudo sabe-se que as células cardíacas expressam altos níveis de receptor de ECA II, podendo explicar o dano tecidual ao miocárdio. Outros mecanismos de lesão cardíaca são descritos, e incluem, estresse secundário a hipoxemia, doença microvascular e injuria indireta, secundária a inflamação sistêmica (FERNADES F, et al., 2020).

Frente a um paciente que teve infecção por SARS-CoV-2 e apresentou piora clínica súbita, é importante considerar complicações cardiovasculares como insuficiência cardíaca exacerbação aguda da insuficiência cardíaca crônica, síndrome coronariana aguda, embolia pulmonar aguda, miocardite, arritmias, mas também afecções pericárdicas, como pericardite (FERNANDES F, et al., 2020; LIU PP, et al., 2020).

A pericardite é um processo inflamatório do pericárdio, que tem inúmeras causas, espacialmente as virais e se apresenta tanto como doença primária quanto secundária, seu curso é em geral benigno e autolimitado, podendo evoluir com derrame ou constrição pericárdica e consequente aumento da sua morbidez (MONTERA MW, et al., 2013). As principais manifestações clínicas da pericardite incluem dor torácica do tipo pleurítica, atrito pericárdico, elevação difusa do segmento ST e derrame pericárdico (FUSCO DR, et al., 2011; MONTERA MW, et al., 2013; FERNANDES F, et al., 2020). 
O diagnóstico clínico é realizado de acordo com a presença de 2 dos 6 critérios (Dor torácica típica, atrito pericárdico, alterações no traçado eletrocardiográfico compatíveis com pericardite, novo ou piora do derrame pericárdico, elevação de marcadores inflamatórios e imagem tomográfica que podem compatível com diagnóstico). O ecocardiograma (ECO) é o exame de imagem que atua como importante ferramenta no diagnóstico, acompanhamento da resposta terapêutica e prognóstico das doenças do pericárdio (MONTERA MW, et al., 2013).

Considerando a pericardite de causa viral a mais comum e tendo em vista o contexto atual da pandemia pela COVID-19 e suas diversas apresentações clínicas e complicações/agravos associados ao comprometimento cardiovascular, faz-se necessário estudos que evidenciem a ocorrência da pericardite no curso clínico da infecção por SARS-CoV-2.

\section{MÉTODOS}

Este estudo possui caráter retrospectivo, observacional, descritivo, do tipo transversal, foi conduzido em uma clínica de cardiologia no Estado do Pará, com coleta de dados em prontuários de pacientes atendidos na referida clínica.

A população do estudo consiste em um grupo de pacientes com história de COVID-19 e pericardite aguda, confirmados por TC e exames laboratoriais, entre os meses de março a junho de 2020. A coleta de dados através de protocolo de pesquisa foi realizada após aprovação pelo Comitê de Ética em Pesquisa (CEP) da Fundação Santa Casa de Misericórdia do Pará (FSCMPA), sob parecer de número 4.429.014.

Os dados retirados dos prontuários foram registrados e organizados de maneira cronológica para a formação do banco de dados e tratados estatisticamente em análise descritiva apropriada, através da frequência absoluta e percentuais. A média e desvio padrão foram utilizados quando necessários.

O banco de dados, a formalização de tabelas e gráficos foi realizada com o software Microsoft Excel e análise dos resultados foi tratada estatisticamente por meio do Teste do Qui-Quadrado (Wilks' G²), com o programa Bioestat 5.0.

\section{RESULTADOS}

Do total de 34 pacientes atendidos com diagnóstico de COVID-19 na clínica cardiológica, entre os meses de março a junho de 2020, 10 pacientes (29.4\%) foram atendidos em março, 15 (44.1\%) atendidos em abril, $5(14.7 \%)$ em maio e $4(11.8 \%)$ em junho (Gráfico 1).

Gráfico 1. Distribuição de casos do COVID-19 atendidos na clínica cardiológica entre os meses de Março e Junho de 2020.

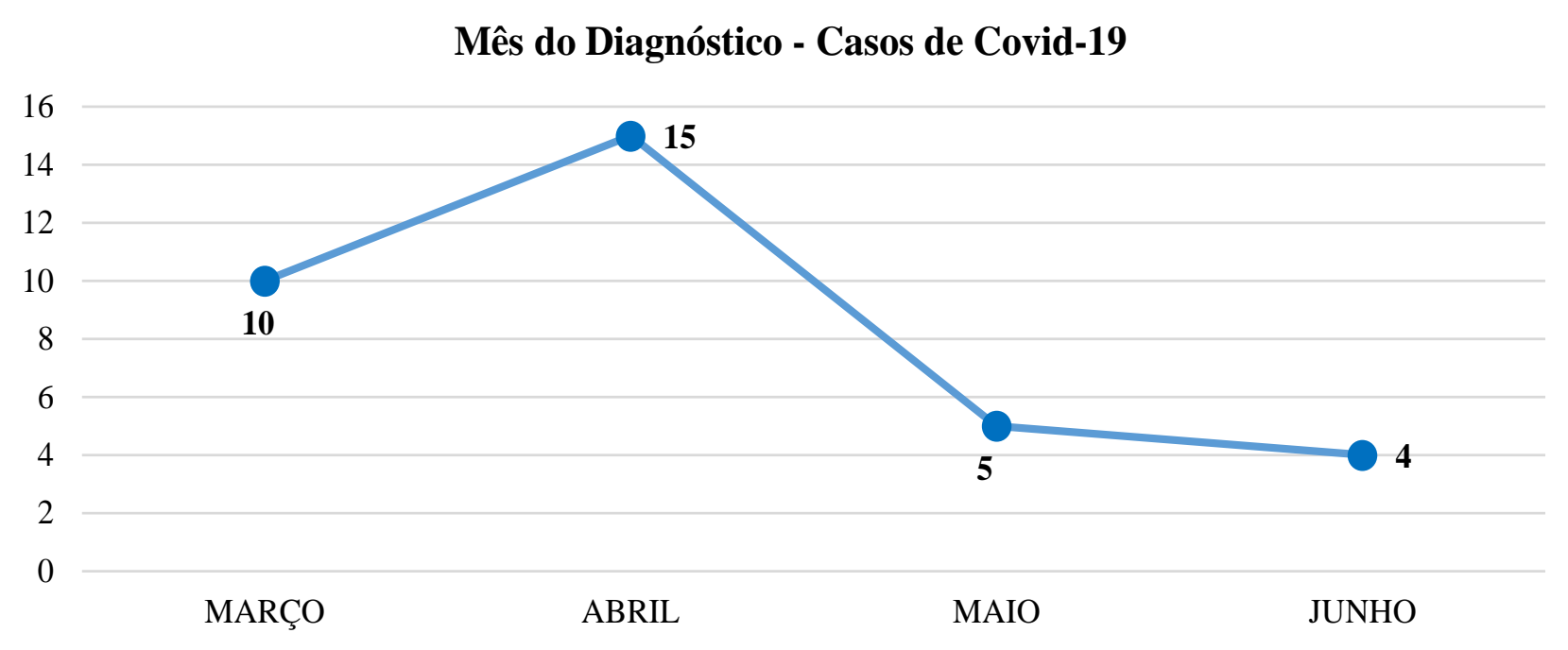

Fonte: Bentes CG, et al., 2021. 
Desse total, 4 pacientes desenvolveram pericardite após infecção pelo SARS-CoV-2, representando uma taxa de incidência de pericardite pós COVID-19 de 11.8\%, que apresentou significância estatística verificada em análise na Tabela 1.

Tabela 1 - Incidência de pericardite pós COVID-19, da clínica cardiológica, no período de março a junho de 2020.

\begin{tabular}{cccc}
\hline Incidência de Pericardite & $\mathbf{n = 3 4}$ & $\%$ & P-Valor $^{\left({ }^{(1)}\right.}$ \\
\hline Sim & 4 & $11.8 \%$ & $0.000^{*}$ \\
Não & 30 & $88.2 \%$ & \\
\hline
\end{tabular}

Nota: (1) Teste Qui-quadrado (Wilks' $\mathrm{G}^{2}$ ) de Pearson para independência (p-valor < 0.05 ). *Valores Significativos; NS - Valores Não Significativos.

Fonte: Bentes CG, et al., 2021.

Dos 4 pacientes observados nesse estudo, 3 são do gênero feminino (75\%) e 1 do gênero masculino (25\%). Nessa amostra, a idade variou de 48 a 80 anos, com uma média de 62 anos ( $\mu=62.25$ DP \pm 13.22 ). Quando realizada análise estatística de gênero e idade para a amostra, foi possível observar que há diferença significativa entre mulheres e homens afetados por pericardite, bem como diferença entre as faixas etárias acometidas (Tabela 2).

Tabela 2 - Distribuição dos casos de pericardite em pacientes com história pregressa de COVID-19, atendidos na clínica cardiológica, no período de março a junho de 2020, segundo gênero e faixa etária.

\begin{tabular}{cccc}
\hline Variável & $\mathbf{n = 4}$ & $\%$ & P-Valor $^{(1)}$ \\
\hline Gênero & & & \multirow{2}{*}{$0.000^{*}$} \\
\hline Feminino & 3 & $75.0 \%$ & \\
Masculino & 1 & $25.0 \%$ & \multirow{2}{*}{$0.002^{*}$} \\
\hline Faixa Etária & & & \\
\hline $48-57$ & 1 & $25.0 \%$ & $50.0 \%$ \\
$58-67$ & 2 & $25.0 \%$ & \\
$78-87$ & 1 & &
\end{tabular}

Nota: ${ }^{(1)}$ Teste Qui-quadrado (Wilks' G²) de Pearson para independência (p-valor < 0.05); *Valores Significativos; NS - Valores Não Significativos.

Fonte: Bentes CG, et al., 2021.

Foram identificados como antecedentes mórbidos pessoais entre os 4 pacientes: hipertensão arterial sistêmica (HAS) em 75\% dos pacientes, diabetes mellitus tipo 2 (DM2), doença coronariana, tabagismo, asma, doença pulmonar obstrutiva crônica (DPOC), hipotiroidismo e acidente vascular cerebral (AVC) em $25 \%$ dos pacientes.

Todos os pacientes com pericardite tiveram diagnóstico prévio da infecção por COVID-19 confirmada pelo exame molecular de RT-PCR para SARS-CoV-2. Verificou-se ainda, que $75 \%$ dos pacientes realizaram Tomografia de tórax como avaliação complementar, apresentando porcentagem de acometimento pulmonar em vidro fosco que variou de 0 a $50 \%$. Contudo, não foi verificado nos registros em prontuário a classificação CO-RADS de nenhum paciente da amostra. Já em relação à gravidade da doença, $75 \%$ dos pacientes apresentaram quadro leve e $25 \%$ moderado.

O intervalo de tempo decorrido entre a confirmação da infecção pelo SARS-CoV-2 e o surgimento da pericardite variou de 15 a 60 dias, apresentando média, em dias, igual a 31.25 ( \pm 17.45$)$. Não houve diferença significativa entre os pacientes que apresentaram diagnóstico até 29 dias e os que apresentaram em 30 dias ou mais (Tabela 3 ).

Em relação ao diagnóstico de pericardite, todos os pacientes fecharam diagnóstico apresentando, entre os critérios, dor torácica sugestiva e derrame pericárdico, $75 \%$ ainda com espessamento do pericárdio associado na imagem obtida pelo ecocardiograma, apresentando significância estatística. Ademais, foi realizado eletrocardiograma em todos os pacientes, porém sem a presença de alterações sugestivas desse diagnóstico na avaliação do exame, também apresentando diferença estatística (Tabela 3). 
Além da dor torácica, presente em $100 \%$ dos pacientes com pericardite, outros sintomas achados incluem palpitações, sudorese, astenia e tremores de extremidades em $25 \%$ dos pacientes, havendo significância estatística entre os sintomas apresentados (Tabela 3).

Em relação ao tratamento da pericardite, observou-se que a totalidade dos pacientes acometidos realizou terapia medicamentosa com colchicina $0,5 \mathrm{mg}$ de $12 / 12$ horas por 3 meses. Os 4 pacientes apresentaram como desfecho clínico a cura da doença aguda do pericárdio, achados que apresentaram significância estatística (Tabela 3). A cura foi confirmada posteriormente, com realização de ECO de controle após o tratamento, no qual se observou a normalidade do exame.

Tabela 3 - Distribuição dos casos de pericardite em pacientes com história pregressa de COVID-19, atendidos na clínica cardiológica, no período de março a junho de 2020, segundo o diagnóstico, clínica, tratamento e desfecho da doença.

\begin{tabular}{|c|c|c|c|}
\hline Variáveis Pericardite & $n=4$ & $\%$ & P-Valor ${ }^{(1)}$ \\
\hline \multicolumn{4}{|l|}{ Eletrocardiograma } \\
\hline Normal & 4 & $100.0 \%$ & \multirow{2}{*}{$0.000^{*}$} \\
\hline Alterado & 0 & $0.0 \%$ & \\
\hline \multicolumn{4}{|l|}{ Ecocardiograma } \\
\hline Derrame pericárdico & 4 & $100.0 \%$ & \multirow{2}{*}{$0.000^{*}$} \\
\hline Sem derrame pericárdico & 0 & $0.0 \%$ & \\
\hline \multicolumn{4}{|l|}{ Sintomas Clínicos } \\
\hline Palpitacões & 1 & $25.0 \%$ & \multirow{6}{*}{$0.000^{*}$} \\
\hline Sudorese & 1 & $25.0 \%$ & \\
\hline Tremores & 1 & $25.0 \%$ & \\
\hline Astenia & 1 & $25.0 \%$ & \\
\hline Cansaço aos médios esforços & 1 & $25.0 \%$ & \\
\hline Dor torácica & 4 & $100.0 \%$ & \\
\hline \multicolumn{4}{|l|}{ Tempo Covid-Pericardite } \\
\hline Até 29 dias & 2 & $50.0 \%$ & \multirow{2}{*}{$1.000 \mathrm{~ns}$} \\
\hline 30 a 60 dias & 2 & $50.0 \%$ & \\
\hline \multicolumn{4}{|l|}{ Tratamento Pericardite } \\
\hline Colchicina $0,5 \mathrm{mg} \mathrm{12/12h}$ & 4 & $100 \%$ & \\
\hline \multicolumn{4}{|l|}{ Tempo de tratamento } \\
\hline Menos de 3 meses & 0 & $0.0 \%$ & \multirow{2}{*}{$0.000^{*}$} \\
\hline 3 meses & 4 & $100.0 \%$ & \\
\hline \multicolumn{4}{|l|}{ Desfecho Clínico } \\
\hline Óbito & 0 & $0.0 \%$ & \multirow{2}{*}{$0.000^{*}$} \\
\hline Melhora/cura & 4 & $100.0 \%$ & \\
\hline
\end{tabular}

Nota: (1) Teste Qui-quadrado (Wilks' $\mathrm{G}^{2}$ ) de Pearson para independência ( $p$-valor < 0.05); *Valores Significativos; NS - Valores Não Significativos.

Fonte: Bentes CG, et al., 2021.

\section{DISCUSSÃO}

Desde o primeiro caso em dezembro de 2019, a COVID-19 espalhou-se rapidamente por todo o mundo, sendo declarada um problema de saúde mundial. Seu patógeno é altamente infeccioso e cursa clinicamente, em sua maioria, com sintomas simples do trato respiratório e complicações como pneumonia e síndrome respiratória aguda (HUANG C, et al., 2020). No âmbito regional, os dados do boletim epidemiológico publicados pela SESPA (2020), demonstraram que o pico de casos diagnosticados com COVID-19 ocorreu em abril de 2020 na Região Metropolitana do estado do Pará. Resultado semelhante ao observado no presente estudo, no qual o maior número de casos de COVID-19 também foi encontrado no referido mês, na clínica onde realizou-se a pesquisa.

Embora tenha sido demonstrado que o SARS-CoV-2 tenha predileção pelos pulmões e através de complicações respiratórias esteja fortemente associada à mortalidade, dados emergentes mostram que o acometimento cardiológico pode estar presente. O envolvimento cardiovascular na COVID-19 é pouco descrito na literatura, com apenas alguns relatos de casos sendo publicados, referindo principalmente a 
ocorrência de afecções coronarianas, do miocárdio e pericárdio. Conforme a doença viral pelo SARS-CoV-2 foi se espalhando globalmente e cada vez mais estudada, relatos de manifestações extrapulmonares foram frequentemente identificadas, porém o envolvimento pericárdico permaneceu raramente relatado (INCIARD RM, et al., 2020; DABBAGH MF, et al., 2020).

Nesse contexto, a ocorrência de pericardite em 4 pacientes com diagnóstico prévio de COVID-19, em um período de 3 meses, determinando a incidência de $11,8 \%$, representa importante achado no contexto da pandemia, uma vez que até apresente data, apenas relatos de casos foram encontrados na literatura revisada.

A pericardite aguda é a inflamação da membrana fibroserosa que recobre o coração, denominada pericárdio, e possui diversas etiologias infecciosas e não infecciosas. Geralmente etiologias virais são as causas mais comuns relacionadas às pericardites causadas por infecção, cursando frequentemente como uma condição autolimitada. No entanto, pouco se sabe sobre o envolvimento cardíaco como complicação da infecção por SARS-CoV-2 (INCIARD RM, et al., 2020). Estudos recentes evidenciam sobre o potencial de lesão cardíaca aguda que ocorre em pacientes infectados com COVID-19. O coronavírus relacionado à síndrome respiratória aguda do Oriente Médio (MERS-CoV) pode causar miocardite e insuficiência cardíaca e ambos, MERS-CoV e SARS-CoV-2, têm patogenicidade semelhante (ZHENG YY, et al., 2020).

Em relação à patogenia, sabe-se que o acometimento cardíaco pelo SARS-CoV-2 pode refletir o processo de replicação e disseminação do vírus através do sangue ou do sistema linfático do trato respiratório. A ação do vírus no pericárdio pode estar associada à ação direta do próprio vírus no tecido por efeito citotóxico e/ou por mecanismo imunomediado. (INCIARD RM, et al., 2020; AGHAGOLI G, et al., 2020).

A liberação excessiva de citocinas é um evento que pode ser observado em várias doenças sistêmicas, a exemplo das infeccisosas, reumatológicas e neoplásicas, as quais podem ocasionar o acometimento pericárdico. O estado hiperinflamatório induzido pela atuação da tempestade de citocinas pode levar à insuficiência de múltiplos órgãos e poderia ser o responsável pelo comprometimento miocárdico e pericárdico na doença, entretanto não podendo ser excluída a possibilidade de parte desse comprometimento ser causada pelo vírus (MAISCH B e DORR R, 2020).

A atuação direta do vírus ocorre através de sua ligação ao receptor de ECA II, presente no endotélio vascular e também no coração, dessa forma, a destruição celular seria mediada pelo receptor de ECA II, impedindo mecanismos contraregulatórios da produção de Angiotensina II, e consequentemente gerando reação inflamatória exacerbada (ASAIED T, et al., 2020). Em decorrência desse mecanismo de ação, o uso de antihipertensivos inibidores de ECA II (IECA) na inibição da infecção por COVID-19 era bastante discutido e controverso, porém em estudo bem recente Semenzato L, et al. (2021), ao analisar 2 milhões de hipertensos entre 18 e 80 anos, por 16 semanas, associou o uso dos IECAs para tratamento da hipertensão, com uma melhor resposta à infecção da COVID-19, ao promover diminuição da hiperinflamação relacionada à infecção pelo SARS-CoV-2, assim como ter melhor resposta antiviral das células, acarretando em risco menor de hospitalização, intubação e morte, quando comparados com outras classes como bloqueadores do receptor de angiotensina (BRA) e bloqueadores dos canais de cálcio (BCC).

No presente estudo foi observado, maior frequência de pericardite no sexo feminino, o que corrobora com o estudo de Fernandes F, et al. (2020) que realizou o levamento de afecções pericárdicas em pacientes infectados por COVID-19 pelo mundo, no qual, de 6 casos encontrados, até o mês de julho de 2020, cinco eram mulheres.

A média de tempo decorrido entre a COVID-19 e o diagnóstico de pericardite, em nosso estudo, foi de aproximadamente 30 dias. Em sua revisão não sistemática, Fernandes $F$, et al. (2020) observou que o diagnóstico e piora clínica por pericardite ocorreram após uma semana da infecção pela COVID-19, aventando que por coincidir com a fase de aumento de citocinas inflamatórias, o desenvolvimento do derrame pericárdico está envolvido com mecamismos autoimunes.

Em contrapartida, de acordo com Inciard RM, et al. (2020), esse envolvimento cardíaco, é um possível fenômeno tardio da infecção respiratória viral da COVID-19. Em um relato de caso realizado na Itália, o paciente descrito apresentou um intervalo de tempo mais tardio até o aparecimento da pericardite, com 
peculiaridades em sua história, como a presença do SARS-CoV-2 e de infiltrado inflamatório com predomínio linfocitário na análise química e citológica do líquido pericárdico, apesar de exames de PCR negativo no sangue e nasofaringe, o que corrobora com o possível efeito direto do vírus nas manifestações cardiológicas (FARINA A, et al., 2020). Em nosso trabalho, não foram encontrados registros de realização de análise do líquido pericárdico em nenhum dos 4 pacientes, porém todos realizaram swab nasofaríngeo positivo para SARS-CoV-2.

Inciard RM, et al. (2020) refere como principal achado, que o envolvimento cardíaco na COVID-19 pode acontecer mesmo na ausência de sinais e sintomas do trato respiratório. Relata, ainda, que o paciente de seu estudo evoluiu com miopericardite e insuficiência cardíaca, sem apresentar história prévia de doença cardiovascular. Nosso estudo também observou uma paciente que não apresentou antecedentes pessoais cardiovasculares, evoluindo com pericardite após 20 dias da vigência da COVID-19, na qual apresentou apenas sintomas respiratórios leves.

Os demais pacientes da amostra, no entanto, possuíam diversas doenças cardiovasculares em seus antecedentes, como HAS, DM2, coronariopatia e AVC. Um estudo de metanálise publicado na China, realizado com pacientes infectados com COVID-19, reportou que $9,7 \%$ dos pacientes tinham diabetes, $16,4 \%$ apresentavam doenças cardiocerebrovasculares e 17,1\% apresentavam hipertensão. Nesse mesmo estudo, embora a prevalência de HAS e DM2 tenha sido considerada a mesma que da população em geral, a prevalência de doenças cardiocerebrovasculares foi considerada alta (LI B, et al., 2020).

Diversos fatores de risco cardíacos podem predizer a susceptibilidade e gravidade da infecção pelo SARSCoV-2 (BANSAL M, 2020). Li B, et al. (2020) também mostraram que pacientes com necessidade de cuidados intensivos, com doenças cerebrovasculares, hipertensão e diabetes, necessitaram de três vezes, duas vezes e duas vezes, respectivamente, mais cuidados intensivos, sugerindo impacto no prognóstico dessas comorbidades.

Portanto, há evidências crescentes de que condições cardiovasculares levam a maior probabilidade de infecção, maior progressão grave da doença e maior risco de mortalidade por COVID-19 (SHI S, et al., 2020). Particularmente, apesar da evidência da pericardite como complicação nos pacientes desse estudo, os antecedentes cardiovasculares encontrados, não interferiram na gravidade da afecção aguda, aventando a possibilidade de tratar-se de evento tardio em relação à infecção pela COVID-19.

Dentro do arsenal diagnóstico, o ecocardiograma tem se mostrado muito útil nas doenças do pericárdio, bem como no acompanhamento da resposta terapêutica e no prognóstico, além de ser utilizado como guia na pericardiocentese. Na pericardite aguda os achados mais comuns envolvem aumento do espessamento pericárdico e derrame pericárdico (MONTERA MW, et al., 2013). Em nossa amostra, todos os pacientes realizaram ECO tanto ao diagnóstico quanto para acompanhamento terapêutico, seguindo as recomendações da I Diretriz Brasileira de Miocardites e Pericardites. Derrame pericárdico foi um achado no ECO de todos os pacientes descritos no presente trabalho, metade classificados com volume discreto e metade com moderado volume. Além disso, na maioria havia espessamento pericárdico associado.

O tratamento da pericardite aguda e recorrente tem como primeira escolha o uso de AINES desde que não apresente contraindicações, contudo o tratamento das afecções do pericárdio em pacientes com infecção atual ou pregressa pelo SARS-CoV-2, ainda encontra-se em discussão. No início da pandemia um grupo francês descreveu o uso de AINES, especialmente o ibuprofeno, para tratamento sintomático de COVID-19, desenvolvendo formas mais graves da doença e período maior de internação dos pacientes, colocando em questão a terapêutica com AINES (ADLER Y, et al., 2015; FERNANDES F, 2020).

$\mathrm{O}$ tratamento de todos os pacientes com pericardite, do presente estudo, foi realizado com o uso de colchicina em seu protocolo terapêutico usual $(0,5 \mathrm{mg}$ de $12 / 12$ horas) para essa infecção aguda, por três meses. Nos últimos anos, a colchicina vem se destacando no tratamento tanto da pericardite aguda quanto da recorrente, com excelentes resultados, devendo ser considerada no tratamento da pericardite com SARSCoV-2. Seus efeitos antiinflamatórios estão relacionados à ruptura da função dos microtúbulos, com parada da divisão celular, resultando na inibição dos neutrófilos e moléculas de adesão que interferem no processo 
inflamatório, assim como na sua exacerbação. Alguns estudos, ainda em andamento, avaliam o uso da colchicina em pacientes com COVID-19, com o objetivo de testar a ação do medicamento em atenuar a inflamação sistêmica e/ou miocárdica (ADLER Y, et al., 2015; FERNANDES F, et al., 2020).

Em relação ao desfecho clínico da pericardite, os pacientes do presente estudo evoluíram com melhora do quadro clínico após o tratamento realizado, sendo considerados curados da doença, após realização de ECO de controle apresentando normalidade. Além disso, nenhum dos pacientes evoluiu com complicações da doença como o tamponamento cardíaco. Ainda em sua revisão não sistemática, Fernandes $F(2020)$, relata que a maioria dos casos se apresentaram com tamponamento cardíaco, uma causa de descompensação clínica de alto risco, mas potencialmente reversível, necessitando alguns de intervenção invasiva com pericardiocentese, quando da ocorrência de instabilidade hemodinâmica.

As manifestações cardíacas na COVID-19, ainda que pouco descritas na literatura, apresentam potencial fator de evolução para complicações clínicas, a exemplo da pericardite aguda, que apesar de autolimitada em sua maioria, pode evoluir com complicações como o tamponamento cardíaco, ou evolução para formas recorrentes e constritivas. Dessa forma, a realização do presente estudo evidencia que a cardiovigilância em pacientes com infecção pregressa ou atual pela COVID-19, é de fundamental importância no que diz respeito ao diagnóstico e tratamento adequados, como foi demonstrado na amostra da clínica cardiológica estudada.

\section{CONCLUSÃO}

Ainda que realizado com uma casuística pequena e isolada em uma clínica cardiológica, o relevante resultado de incidência de pericardite, como complicação tardia relacionada à COVID-19, foi um achado importante em nosso estudo, permitindo a expansão do conhecimento dentro do contexto desafiador da pandemia e assegurando as manifestações cardíacas como uma das principais e mais relevantes, manifestações extrapulmonares da COVID-19. Tal resultado instiga-nos a refletir o quanto uma variedade de outras manifestações cardiovasculares relacionadas à infecção pelo SARS-CoV-2, permanecem pouco relatadas e estudadas, quando dos raros relatos encontrados na literatura. Por fim, a partir dos resultados encontrados na amostra estudada, também se concluiu que a pericardite pós COVID-19 manteve seu padrão autolimitado, uma vez diagnosticada e tratada adequadamente.

\section{AGRADECIMENTOS E FINANCIAMENTO}

Os autores agradecem os funcionários da Clínica CardioDiagnóstico que auxiliaram de todas as formas as coletas de dados necessárias para realização do presente trabalho.

\section{REFERÊNCIAS}

1. ADLER Y, et al. 2015 ESC Guidelines for the diagnosis and management of pericardial diseases: the Task Force for the Diagnosis and Management of Pericardial Diseases of the European Society of Cardiology (ESC) Endorsed by: The European Association for Cardio-Thoracic Surgery (EACTS). European heart journal, 2015; 36(42): $2921-2964$.

2. AGHAGOLI G, et al. Cardiac involvement in COVID-19 patients: Risk factors, predictors, and complications: $A$ review. Journal of cardiac surgery, 2020; 35 (6): 1302-1305.

3. BANSAL M. Cardiovascular disease and COVID-19. Diabetes \& Metabolic Syndrome: Clinical Research \& Reviews, 2020.

4. SESPA. Secretaria de Saúde Pública. Boletim Epidemiológico COVID-19. 2020. Disponível em: http://www.saude.pa.gov.br/wp-content/uploads/2020/07/Boletim-Epidemiol\%C3\%B3gico-COVID-19_10.07.2020.pdf. Acesso em: 19 jan. 2021.

5. DABBAGH MF, et al. Cardiac tamponade secondary to COVID-19. Case Reports, 2020; 2 (9): 1326-1330.

6. FARINA A, et al. SARS-CoV-2 detection in the pericardial fluid of a patient with cardiac tamponade. European journal of internal medicine, 2020; 76: 100.

7. FERNANDES F, et al. Afecções Pericárdicas em Pacientes com COVID-19: Uma Possível Causa de Deterioração Hemodinâmica. Arquivos Brasileiros de Cardiologia, 2020; 115(3): 569-573.

8. FIGUEIREDO NETO JA DE, et al. Doença de Coronavírus-19 e o Miocárdio. Arquivos Brasileiros de Cardiologia, 2020; 114(6): 1051-1057. 
9. FUSCO DR, et al. Pericardite aguda e crônica. Rev. Soc. Cardiol. Estado de Säo Paulo, 2011: 30-37.

10. GUZIK TJ, et al. COVID-19 and the cardiovascular system: implications for risk assessment, diagnosis, and treatment options. Cardiovascular research, 2020.

11. HUANG C, et al. Clinical features of patients infected with 2019 novel coronavirus in Wuhan, China. The lancet, 2020; 395 (10223): 497-506.

12. INCIARDI RM, et al. Cardiac involvement in a patient with coronavirus disease 2019 (COVID-19). JAMA cardiology, 2020; 5(7): 819-824.

13. LI B, et al. Prevalence and impact of cardiovascular metabolic diseases on COVID-19 in China. Clinical Research in Cardiology, 2020, 109(5): 531-538.

14. LIU PP, et al. The science underlying COVID-19: implications for the cardiovascular system. Circulation, 2020.

15. MAISCH B, DÖRR R. COVID-19-What we know and what we need to know: There are more questions than answers. 2020.

16. MERAD M, MARTIN JC. Pathological inflammation in patients with COVID-19: a key role for monocytes and macrophages. Nature Reviews Immunology, 2020: 1-8.

17. MITRANI RD, et al. COVID-19 cardiac injury: Implications for long-term surveillance and outcomes in survivors. Heart rhythm, 2020.

18. MONTERA MW, et al. I Diretriz Brasileira de Miocardites e Pericardites. Arquivos Brasileiros de Cardiologia, 2013; 100(4): 01-36.

19. MOORE JB, JUNE CH. Cytokine release syndrome in severe COVID-19. Science, 2020; 368(6490): $473-474$.

20. SEMENZATO L, et al. Antihypertensive Drugs and COVID-19 Risk. Hypertension, 2021; 77(3): 833-842.

21. SHI S, et al. Association of cardiac injury with mortality in hospitalized patients with COVID-19 in Wuhan, China. JAMA cardiology, 2020; 5(7): 802-810.

22. SOUSA MRN, et al. Patogênese e perspectivas de tratamento da Covid- 19: uma revisão. Research, Society and Development, 2020; 9(7): e05973730-e05973730.

23. YUKI K, et al. COVID-19 pathophysiology: A review. Clinical immunology, 2020: 108427.

24. ZHENG YY, et al. COVID-19 and the cardiovascular system. Nature Reviews Cardiology, 2020; 17(5): $259-260$. 